\title{
DOTS improves treatment outcomes and service coverage for tuberculosis in South Ethiopia: a retrospective trend analysis Estifanos B Shargie*1,2 and Bernt Lindtjørn ${ }^{1}$
}

Address: ${ }^{1}$ University of Bergen Centre for International Health, Armauer Hansens Hus, N-5021 Bergen, Norway and ${ }^{2}$ Southern Nations, Nationalities and Peoples' Regional State Health Bureau, P. O. Box 149, Awassa, Ethiopia

Email: Estifanos B Shargie* - estifanos.shargie@rasmus.uib.no; Bernt Lindtjørn - bernt.lindtjorn@cih.uib.no

* Corresponding author

Published: 06 June 2005

BMC Public Health 2005, 5:62 doi:10.1 I86/147|-2458-5-62
Received: 12 January 2005

Accepted: 06 June 2005

This article is available from: http://www.biomedcentral.com/I47I-2458/5/62

(c) 2005 Shargie and Lindtjørn; licensee BioMed Central Ltd.

This is an Open Access article distributed under the terms of the Creative Commons Attribution License (http://creativecommons.org/licenses/by/2.0), which permits unrestricted use, distribution, and reproduction in any medium, provided the original work is properly cited.

\begin{abstract}
Background: DOTS as a strategy was introduced to the tuberculosis control programme in Southern region of Ethiopia in 1996. The impact of the programme on treatment outcomes and the trend in the service coverage for tuberculosis has not been assessed ever since. The aim of the study was to assess trends in the expansion of DOTS and treatment outcomes for tuberculosis in Hadiya zone in Southern Ethiopia.
\end{abstract}

Methods: 19,97I tuberculosis patients registered for treatment in $4 \mathrm{I}$ treatment centres in Hadiya zone between 1994 and 2001 were included in the study. The data were collected from the unit tuberculosis registers. For each patient, we recorded information on demographic characteristics, treatment centre, year of treatment, disease category, treatment given, follow-up and treatment outcomes. We also checked the year when DOTS was introduced to the treatment centre.

Results: Population coverage by DOTS reached $75 \%$ in 200 I, and the proportion of patients treated with short course chemotherapy increased from $7 \%$ in 1994 to $97 \%$ in 200 I. Treatment success for smear-positive tuberculosis rose from $38 \%$ to $73 \%$ in 2000 , default rate declined from $38 \%$ to $18 \%$, and treatment failure declined from $5 \%$ to $1 \%$. Being female patient, age 15-24 years, smear positive pulmonary tuberculosis, treatment with short course chemotherapy, and treatment at peripheral centres were associated with higher treatment success and lower defaulter rates.

Conclusion: The introduction and expansion of DOTS in Hadiya has led to a significant increase in treatment success and decrease in default and failure rates. The smaller institutions exhibited better treatment outcomes compared to the larger ones including the zonal hospital. We identified many patients with missing information in the unit registers and this issue needs to be addressed. Further studies are recommended to see the impact of the programme on the prevalence and incidence of tuberculosis.

\section{Background}

Ethiopia stands among the world's top 22 tuberculosis (TB) high-burden countries, with an estimated annual incidence of $250 \mathrm{~TB}$ cases $/ 10^{5}$ population [1,2]. In the
Southern Ethiopia Regional State (SNNPRS), TB is among the leading causes for sickness and death [3]. As in many other resource-constrained settings, treatment outcomes for tuberculosis have not been satisfactory [4], mainly due 
to poor treatment compliance and low coverage of short course chemotherapy (SCC). Delays in the diagnosis and treatment initiation, the devastating HIV/AIDS epidemic and the potential threat of anti-tuberculosis drug resistance represent serious threats to the TB control effort in the region. The HIV co-infection among TB patients in the region is estimated at $19 \%$ [5].

Directly Observed Treatment, Short-course (DOTS) was introduced in the region in 1996. However, the impact of DOTS and its effectiveness in the regional context has not been assessed yet. Global reviews [6] and reports from Asian, African and East European countries [7-9] have favourable implications for the DOTS strategy. On the contrary, some randomised controlled studies have failed to establish the superiority of the most notable component of this strategy, direct observation of treatment, over the conventional non-observed treatment in improving treatment outcomes [10,11]. Nevertheless, DOTS is widely accepted and practised, with some TB high-burden countries achieving almost full coverage of their populations [2]. DOTS is equally effective in curing TB patients with HIV co-infection $[9,12]$ and hence, its importance in the era of the HIV/AIDS epidemic.

With this study, we aimed to assess the results and trends in the expansion of DOTS in Hadiya Zone, Southern Ethiopia.

\section{Methods \\ Study setting}

We did the study in Hadiya zone, Southern Ethiopia (see map in Figure 1), with a population of 1.2 million $[13,14]$. In addition to fifteen diagnostic centres, five health centres and 21 health stations provide treatment for TB patients referred or transferred from the diagnostic centres, thus making the number of treatment centres 41 . All treatment units have standard Unit Registers from the National Tuberculosis and Leprosy Control Programme (TLCP). TB drugs used in a combination of two or more in the region included isoniazid $(H)$, rifampicin $(R)$, pyrazinamide $(Z)$, ethambutol $(E)$, streptomycin $(S)$ and thioacetazone $(\mathrm{T})$.

The TB and Leprosy control programs were run as two separate vertical programs funded and managed mainly by the All African Leprosy and Rehabilitation Training Centre (ALERT) until the end of 1996 when the National and Regional TLCP took over the responsibility. The zonal TLCP gets administrative and technical support from the regional TLCP, and monitors the activities in the diagnostic and treatment centres through its seven woreda (district) offices. The woreda health offices have standard district TB registers from the National TLCP, and are responsible for the distribution of drugs and reagents, supervision of day to day activities in the treatment units, collection of slides for quality control and record keeping. Quarterly reports on cases detected and treatment outcomes from the woredas are gathered at the zonal level and sent to the regional TLCP.

In 1994, only Hossana Hospital provided unobserved SCC for critically sick smear-positive pulmonary TB $(\mathrm{PTB}+)$, miliary $\mathrm{TB}$ and tuberculosis meningitis cases. DOTS was initiated in 1996 in two health facilities and gradually expanded, first to the health centres in all woredas and then to the health stations. Details of treatment regimens for various categories of patients under DOTS are given in the NTLCP manual [15]. In brief, all new patients were treated with SCC (two months on RHZ +/E or S, followed by 6 months on EH or RH), and were required to take their medications under direct supervision by the health workers at least during the intensive phase of treatment. During this phase, only critically ill patients were hospitalised, while the rest received treatment on ambulatory basis. Re-treatment cases were treated with SERHZ for 2 months, ERHZ for 1 month and ERH for 5 months. In non-DOTS areas treatment with SCC was limited to critically sick PTB+, TB meningitis and miliary TB cases while others received LCC (two months on $\mathrm{EH}+/-\mathrm{S}$, followed by 10 months on EH; TH had also been used as an alternative to $\mathrm{EH}$ until its recent withdrawal).

\section{Design and data collection}

This is a retrospective trend analysis. The Unit Registers reviewed contain basic information such as patient's age, sex, address, category, TB type, drug regimen, date treatment started, treatment follow-up, follow-up sputum result and treatment outcomes.

We visited all 41 TB treatment centres during July-November 2002. We checked when DOTS was initiated in each health institution and looked for the availability of TB drugs and reagents during the time of visit. We then reviewed the Unit Registers and entered the data to the computer. At three peripheral treatment centres where the unit registers could not be found, the respective district TB registers were reviewed. For each TB case, we copied the data from the registers to a computer program, SPSS for windows [16], according to the standard definitions of the National TLCP [15] on disease classification, patient categories, treatment regimens and treatment outcomes. To ensure the quality of data entered into the computer database, two people independently cross-checked each entry.

\section{Data analysis and Statistics}

The data were analysed using SPSS for Windows version 11.0 [16]. For categorical data, we used proportions with $95 \%$ confidence intervals, Odds ratio and Chi-square test 
Table I: General Characteristics of the study subjects $(n=$ (997I), 1994-200I

\begin{tabular}{|c|c|c|}
\hline Characteristics & Number & Percent \\
\hline \multicolumn{3}{|l|}{ Age group (years) } \\
\hline $0-14$ & 3356 & 16.8 \\
\hline $15-24$ & 6262 & 31.4 \\
\hline $25-34$ & 5366 & 26.9 \\
\hline $35-44$ & 2706 & 13.5 \\
\hline $45-54$ & 1248 & 6.2 \\
\hline $55-64$ & 501 & 2.5 \\
\hline$\geq 65$ & 220 & I.I \\
\hline Unknown & 312 & 1.6 \\
\hline \multicolumn{3}{|l|}{ Sex } \\
\hline Male & 11138 & 55.8 \\
\hline Female & 8819 & 44.2 \\
\hline Not mentioned & 14 & 0.1 \\
\hline \multicolumn{3}{|l|}{ Patient Category } \\
\hline New & 18687 & 93.6 \\
\hline Transferred-in & 558 & 2.8 \\
\hline Return after default & 273 & 1.4 \\
\hline Failure & 142 & 0.7 \\
\hline Relapse & 139 & 0.6 \\
\hline Unknown & 172 & 0.9 \\
\hline \multicolumn{3}{|l|}{ TB Classification } \\
\hline Pulmonary positive & 9232 & 46.2 \\
\hline Pulmonary negative & 4225 & 21.2 \\
\hline Extra-pulmonary & 6453 & 32.3 \\
\hline Unknown & 61 & 0.3 \\
\hline \multicolumn{3}{|l|}{ Treatment Centre } \\
\hline Hossana Hospital & 5362 & 26.8 \\
\hline Lemmo district health facilities & 4011 & 20.1 \\
\hline Shashogo district health facilities & 1208 & 6.0 \\
\hline Misha district health facilities & 2660 & 13.3 \\
\hline Gibe district health facilities & 1984 & 9.9 \\
\hline Soro district health facilities & 2313 & 11.6 \\
\hline Duna district health facilities & 216 & 1.1 \\
\hline Badewacho district health facilities & 2217 & 11.1 \\
\hline
\end{tabular}

to compare different groups. Multivariate analysis using logistic regression model was used to analyse the association between treatment outcomes and potential predictor variables. We set the level of statistical significance at $5 \%$.

Treatment outcomes were analysed for the years 19942000 because a considerable proportion of patients registered during 2001 were still on treatment during the time of data collection. (The Ethiopian fiscal year goes from July to June. Fiscal year 2001, for example, goes from July 2001 through June 2002, and our study was commenced in July 2002). Patients with unrecorded treatment outcome were analysed as defaulters.

\section{Results \\ Patient registration and case notification}

A total of 19971 tuberculosis patients, 11138 (55.8\%) males and 8819 (44.2\%) females were registered between 1994 and 2001 with the mean (SD) age of 25.6 (13.2) years. Forty-six percent $(n=9232)$ of the patients were PTB+ cases. Overall, 18687 (93.6\%) patients were registered as new cases, $558(2.8 \%)$ as transferred in, 273 $(1.4 \%)$ as return after default, $142(0.7 \%)$ as failure and $139(0.7 \%)$ as relapse cases whereas patient category was not recorded for $172(0.9 \%)$ cases. Table 1 shows the general characteristics of the patients.

The proportion of women among TB patients registered for treatment remained at the range of $40-45 \%$ across the years and at the range of $43-47 \%$ across the age groups below 45 . However, among patients older than 45 years, the proportion of women was significantly lower (31\%; 95\%CI 29-33) compared to those below 45 (46\%; 95\%CI 45-47).

Table 2: Trends in case notification of smear-positive pulmonary TB, Hadiya Zone, 1994-200I

\begin{tabular}{|c|c|c|c|c|}
\hline Year & Zonal Population* & $\begin{array}{l}\text { New PTB+ cases Reported } \\
(\mathrm{n}=8558)\end{array}$ & $\begin{array}{l}\text { Case Notification/ } 10^{5} \\
\text { persons/year }\end{array}$ & $\begin{array}{c}\text { Proportion of estimated } \\
\text { PTB+ incident cases } \\
\text { notified, \%** }\end{array}$ \\
\hline 1994 & 1004000 & 512 & 49 & 45.2 \\
\hline 1995 & 1070160 & 1025 & 96 & 87.9 \\
\hline 1996 & 1101195 & 1162 & 106 & 96.8 \\
\hline 1997 & 1133129 & 946 & 84 & 76.6 \\
\hline 1998 & II 65990 & 1277 & 110 & 100.5 \\
\hline 1999 & II99804 & 1510 & 126 & II5.5 \\
\hline 2000 & 1234598 & 1267 & 103 & 94.2 \\
\hline $2001 \S$ & $1174 \mid 18$ & 858 & 73 & 67.0 \\
\hline
\end{tabular}

*Projected from the 1994 Population and Housing Census, CSA [13]

**Estimated Incidence of PTB+ = 109/105 persons/year based on the WHO estimates of the Global Burden of Tuberculosis for 1997 [I]

$\S$ Population adjusted as an area with about 70-80 thousand population was taken away from Hadiya to the neighbouring Silti zone between years 2000 and 2001

PTB+: smear-positive pulmonary tuberculosis 
Table 3: Trend in treatment regimens for the different categories of patients $(n=19970) * *$

\begin{tabular}{|c|c|c|c|c|c|c|c|c|}
\hline Category & 1994 n (\%) & 1995 n (\%) & 1996 n (\%) & 1997 n (\%) & 1998 n (\%) & 1999 n (\%) & 2000 n (\%) & 2001 n (\%) \\
\hline All TB cases & 1106 & 2201 & 2895 & 2742 & 3388 & 3253 & 2435 & 1950 \\
\hline SCC & $73(7)$ & $311(14)$ & $288(10)$ & $471(17)$ & $927(27)$ & $|88|(58)$ & $2085(86)$ & $1883(97)$ \\
\hline LCC & 1011 (91) & 1684 (77) & $2360(82)$ & 2097 (77) & $2292(68)$ & $1302(40)$ & $304(12)$ & $20(1)$ \\
\hline No record & $22(2)$ & $206(9)$ & $247(8)$ & $174(6)$ & $169(5)$ & $70(2)$ & $46(2)$ & $47(2)$ \\
\hline New PTB+ & 512 & 1025 & 1162 & 946 & 1277 & 1510 & 1267 & 858 \\
\hline SCC & $9(2)$ & III (II) & $94(8)$ & $232(24)$ & $537(42)$ & $1170(78)$ & $1187(94)$ & 837 (97) \\
\hline LCC & $496(97)$ & $780(76)$ & $938(81)$ & $611(65)$ & $660(52)$ & $32 I(2 I)$ & $68(5)$ & $7(I)$ \\
\hline No record & $7(1)$ & $134(13)$ & $130(11)$ & $103(11)$ & $80(6)$ & $19(1)$ & $12(1)$ & $14(2)$ \\
\hline New PTB- & 258 & 385 & 402 & 382 & 807 & 772 & 487 & 393 \\
\hline SCC & $12(5)$ & $66(17)$ & $90(22)$ & $109(28)$ & $225(28)$ & $395(51)$ & $402(83)$ & $379(96)$ \\
\hline LCC & $242(94)$ & $286(74)$ & $248(62)$ & 251 (66) & $543(67)$ & 362 (47) & $73(15)$ & - \\
\hline No record & $4(1)$ & $33(9)$ & $64(16)$ & $22(6)$ & $39(5)$ & $15(2)$ & $12(2)$ & $14(4)$ \\
\hline New EPTB & 257 & 668 & 1177 & 1176 & 1124 & 721 & 528 & 570 \\
\hline SCC & $19(8)$ & $73(11)$ & $57(5)$ & $70(6)$ & $97(9)$ & $197(27)$ & $385(73)$ & $559(98)$ \\
\hline LCC & $232(90)$ & $565(85)$ & $1081(92)$ & $1067(91)$ & $993(88)$ & $513(71)$ & $138(26)$ & $6(1)$ \\
\hline No record & $6(2)$ & $30(4)$ & $39(3)$ & $39(3)$ & $34(3)$ & $11(2)$ & $5(1)$ & $5(1)$ \\
\hline Relapse & 8 & 13 & 21 & 29 & 15 & 19 & 19 & 15 \\
\hline $\mathrm{SCC}$ & $2(25)$ & $10(77)$ & $12(57)$ & $21(72)$ & $13(87)$ & $17(90)$ & $19(100)$ & $14(93)$ \\
\hline LCC & $4(50)$ & $2(15)$ & $9(43)$ & $6(21)$ & $2(13)$ & $2(10)$ & - & - \\
\hline No record & $2(2)$ & I (8) & - & $2(7)$ & - & - & - & I (7) \\
\hline After default & 35 & 56 & 27 & 45 & 33 & 25 & 21 & 31 \\
\hline SCC & $5(14)$ & $14(25)$ & $15(56)$ & $20(45)$ & $15(46)$ & $21(84)$ & $17(81)$ & $30(97)$ \\
\hline LCC & $30(86)$ & $39(70)$ & $I I(4 I)$ & $23(5 \mathrm{I})$ & $18(54)$ & $4(16)$ & $4(19)$ & \\
\hline No record & - & $3(5)$ & I (3) & $2(4)$ & - & - & - & I (3) \\
\hline After failure & 28 & 38 & 17 & 17 & 13 & 19 & 8 & 2 \\
\hline SCC & $26(93)$ & $35(92)$ & $15(88)$ & $13(77)$ & II (85) & $14(74)$ & $8(100)$ & $2(100)$ \\
\hline LCC & $2(7)$ & $3(8)$ & $2(12)$ & $4(23)$ & $2(15)$ & $5(26)$ & - & - \\
\hline No record & - & - & - & - & - & - & - & - \\
\hline Transferred in & 6 & 3 & 65 & 131 & 89 & 120 & 86 & 57 \\
\hline SCC & - & - & $3(5)$ & $3(2)$ & $22(25)$ & $43(36)$ & $53(62)$ & $38(67)$ \\
\hline LCC & $5(83)$ & $2(67)$ & $55(84)$ & $124(95)$ & $58(65)$ & $57(47)$ & $19(22)$ & $7(12)$ \\
\hline No record & I (I7) & I (33) & $7(11)$ & $4(3)$ & $9(10)$ & $20(17)$ & $14(16)$ & $12(2 \mid)$ \\
\hline Missing info & 2 & 13 & 17 & 10 & 20 & 46 & 13 & 29 \\
\hline SCC & - & $2(15)$ & I (5) & $3(30)$ & $3(15)$ & II (24) & $9(69)$ & $29(100)$ \\
\hline LCC & - & $7(54)$ & $12(71)$ & $6(60)$ & II (55) & $30(65)$ & $2(16)$ & - \\
\hline No record & 2 & $4(31)$ & $4(24)$ & $I(10)$ & $6(30)$ & $5(11)$ & $2(15)$ & - \\
\hline
\end{tabular}

* Percentages are out of the total cases registered in the same category during the year.

$\mathrm{PTB}+=$ smear-positive pulmonary tuberculosis; $\mathrm{PTB}-=$ smear-negative pulmonary tuberculosis; $\mathrm{EPTB}=$ extra-pulmonary tuberculosis $\mathrm{SCC}=$ short course chemotherapy ( 2 months on $\mathrm{RHZ}+/-\mathrm{E}$ or $\mathrm{S}$, followed by 6 months on $\mathrm{EH}$ or $\mathrm{RH}$ )

$\mathrm{LCC}=$ long course chemotherapy ( 2 months on $\mathrm{EH}+/-\mathrm{S}$, followed by 10 months on $\mathrm{EH}$; $\mathrm{TH}$ had also been used as an alternative to $\mathrm{EH}$ until its recent withdrawal).

$\mathrm{R}=$ Rifampicin,; $\mathrm{H}=$ Isoniazid; $\mathrm{Z}$ = Pyrazinamide; $\mathrm{E}=$ Ethambutol; $\mathrm{S}=$ Streptomycin; $\mathrm{T}$ = Thioacetazone.

Among new cases, 46\% $(\mathrm{n}=8557)$ were PTB+ patients. Except for the years 1997 (38\%; 95\%CI 36-40) and 2000 (56\%; 95\%CI 54-58), this proportion showed little variations in the range of $40-50 \%$. Table 2 shows a trend in the case notification of $\mathrm{PTB}+$ over the study period. The proportion of expected $\mathrm{PTB}+$ incident cases notified increased from $45 \%$ in 1994 to $116 \%$ in 1999 , and declined to $67 \%$ in 2001 . 
Table 4: Trends in the follow-up smear results for new smear positive pulmonary tuberculosis

\begin{tabular}{|c|c|c|c|c|c|c|}
\hline & \multicolumn{2}{|l|}{$2^{\text {nd }}$ month } & \multicolumn{2}{|l|}{$5^{\text {th }}$ month } & \multicolumn{2}{|l|}{ End of treatment } \\
\hline & Examined for AFB (\% total) & $s m+$ & Examined for AFB (\% total) & $\mathrm{Sm}+$ & Examined for AFB (\% total) & $s m+$ \\
\hline \multicolumn{7}{|c|}{ Cases on SCC } \\
\hline 1994 & $6(67)$ & 0 & $5(56)$ & 0 & $3(33)$ & 0 \\
\hline 1995 & $79(7 I)$ & $18(23 \%)$ & $53(48)$ & $4(8 \%)$ & $33(30)$ & 0 \\
\hline 1996 & $68(72)$ & $15(22 \%)$ & $38(40)$ & $2(5 \%)$ & $25(27)$ & 0 \\
\hline 1997 & $155(67)$ & $14(9 \%)$ & $94(4 I)$ & 0 & $74(32)$ & $2(3 \%)$ \\
\hline 1998 & $342(64)$ & $24(7 \%)$ & $183(34)$ & 0 & $140(26)$ & 0 \\
\hline 1999 & $824(70)$ & $52(6 \%)$ & $411(35)$ & $7(2 \%)$ & $365(31)$ & $2(0.5 \%)$ \\
\hline 2000 & $817(69)$ & $43(5 \%)$ & $439(37)$ & $9(2 \%)$ & $316(27)$ & $2(1 \%)$ \\
\hline $200 \mid \S$ & $527(63)$ & $13(3 \%)$ & I7| (20) & I $(0.6 \%)$ & $121(14)$ & 0 \\
\hline \multicolumn{7}{|c|}{ Cases on LCC } \\
\hline 1994 & $330(66)$ & $45(14 \%)$ & $154(3 \mid)$ & $19(12 \%)$ & $86(18)$ & $5(6 \%)$ \\
\hline 1995 & $508(65)$ & $75(15 \%)$ & $354(45)$ & $15(4 \%)$ & $179(23)$ & $4(2 \%)$ \\
\hline 1996 & $564(60)$ & $59(11 \%)$ & $385(4 I)$ & $7(2 \%)$ & $223(24)$ & $4(2 \%)$ \\
\hline 1997 & $302(49)$ & $15(5 \%)$ & $188(31)$ & $5(3 \%)$ & $86(14)$ & $3(4 \%)$ \\
\hline 1998 & $263(40)$ & $19(7 \%)$ & 127 (19) & $7(6 \%)$ & $80(12)$ & $2(3 \%)$ \\
\hline 1999 & $182(57)$ & 7 (4\%) & $86(27)$ & I (I\%) & $68(2 I)$ & $2(3 \%)$ \\
\hline 2000 & $46(68)$ & I (2\%) & $9(13)$ & 0 & $4(6)$ & 0 \\
\hline $2001 \S$ & $2(29)$ & I & $2(29)$ & 0 & I (14) & 0 \\
\hline \multicolumn{7}{|c|}{ Unknown regimen } \\
\hline 1994 & 0 & 0 & $2(29)$ & I & $2(29)$ & 0 \\
\hline 1995 & $9(7)$ & 0 & $4(3)$ & 0 & $3(2)$ & 0 \\
\hline 1996 & $25(19)$ & 0 & $9(7)$ & 0 & $3(2)$ & 0 \\
\hline 1997 & $23(22)$ & 0 & II (II) & 0 & $3(3)$ & 0 \\
\hline 1998 & $27(34)$ & 0 & $19(24)$ & 0 & $5(6)$ & 0 \\
\hline 1999 & $4(21)$ & 0 & I (5) & 0 & I (5) & 0 \\
\hline 2000 & $4(33)$ & 0 & $1(8)$ & 0 & 0 & 0 \\
\hline $2001 \S$ & $5(36)$ & 0 & 17) & 0 & $2(14)$ & 0 \\
\hline
\end{tabular}

sm+ = sputum smear positive for acid-fast bacilli out of examined.

$\mathrm{SCC}=$ short course chemotherapy; $\mathrm{LCC}=$ long course chemotherapy; AFB = acid-fast bacilli.

§Follow-up smears for the $5^{\text {th }}$ month and end of treatment were not complete for the year $200 \mathrm{I}$ as a considerable number of patients were still on treatment during the time of data collection.

\section{Treatment given}

Forty percent $(\mathrm{n}=7919)$ of patients received SCC. Treatment regimen was not recorded for $981(5 \%)$ cases. Table 3 presents trend in treatment regimens across the years by $\mathrm{TB}$ classification. The proportion of patients treated with SCC increased from $7 \%$ in 1994 to $58 \%$ in 1999 , and $97 \%$ in $2001\left(\chi_{\text {trend }}^{2} \mathrm{p}<0.001\right)$.

During 1994-1997, more PTB- patents received SCC compared to PTB+ patients $(19 \%$ vs. $12 \% ; \mathrm{p}<0.05)$ and ЕРTB patients $(19 \%$ vs. $7 \%$; $<<0.05)$ among new cases of TB (Table 3). This trend was reversed after 1997 when more PTB+ patients (76\%) were put on SCC, followed by PTB$(57 \%)$ and ЕРТВ $(42 \%)$. There was no significant difference in the treatment regimens across the age groups and between the two sexes.

\section{Follow-up}

Of the 8557 new PTB+ cases, sputum examination was repeated at the end of two months treatment for $60 \%$ (n $=5112)$, and $7.8 \%(n=401)$ remained positive for AFB. At the end of five months treatment, 2747 (32\%) patients had their sputum examined for AFB, and 78 (2.8\%) remained smear-positive. 1823 (21\%) patients had their sputum examined for AFB at (a month prior to) treatment completion, and $1.4 \%(\mathrm{n}=26)$ remained smear-positive. There has been a continuous and significant decline over time in the sputum positivity at two months irrespective of treatment regimen (Table 4).

Sputum positivity at the completion of fifth month treatment remained at the range of $0-2 \%$ for those on SCC, with the exception of 1995 and 1996 that exhibited 8\% and $5 \%$ respectively. For those on LCC, smear positivity at $5^{\text {th }}$ month decreased from $12 \%$ in 1994 to $1 \%$ in 1999 . Overall, patients on LCC contributed more to the $5^{\text {th }}$ 
Table 5: Treatment outcomes for different categories of patients on short and long course regimens $(n=16943)$

\begin{tabular}{|c|c|c|c|c|c|c|c|}
\hline & \multicolumn{3}{|c|}{ New cases } & \multirow[b]{2}{*}{ Relapse (\%) } & \multirow[b]{2}{*}{ After default (\%) } & \multirow[b]{2}{*}{ After failure (\%) } & \multirow[b]{2}{*}{ Transferred in (\%) } \\
\hline & PTB+ (\%) & PTB- (\%) & ЕРТВ (\%) & & & & \\
\hline \multicolumn{8}{|l|}{ Patients on SCC } \\
\hline Treatment success & $2166(65)$ & $475(37)$ & $436(48)$ & $62(66)$ & $49(46)$ & $65(53)$ & $79(64)$ \\
\hline Default* & $726(22)$ & $483(37)$ & $320(36)$ & $13(14)$ & $45(42)$ & $31(25)$ & $33(26)$ \\
\hline Failure & $25(0.7)$ & - & - & $2(2)$ & $I(1)$ & $6(5)$ & $3(2)$ \\
\hline Death & $117(3.5)$ & $72(5)$ & $24(3)$ & II (I2) & $5(5)$ & $2(1)$ & $2(2)$ \\
\hline Transferred out & $306(9)$ & $269(21)$ & $118(13)$ & $6(6)$ & 7 (7) & $18(15)$ & $7(6)$ \\
\hline \multicolumn{8}{|l|}{ Patients on LCC } \\
\hline Treatment success & $1986(51)$ & 772 (39) & $1932(42)$ & $14(56)$ & $32(25)$ & II (6I) & $189(59)$ \\
\hline Default* & 1365 & $972(48)$ & $1922(42)$ & $8(33)$ & $79(61)$ & $2(11)$ & $24(8)$ \\
\hline Failure & $66(2)$ & - & - & I (4) & $2(2)$ & $3(17)$ & I $(0.3)$ \\
\hline Death & $107(3)$ & $57(3)$ & $37(1)$ & $2(8)$ & $2(2)$ & I (5) & $7(2)$ \\
\hline Transferred out & $350(9)$ & $204(10)$ & $697(15)$ & - & $14(11)$ & $I(6)$ & $3(1)$ \\
\hline
\end{tabular}

* Patients with missing treatment outcome information were assumed to have defaulted and analysed as defaulters.

PTB $+=$ smear positive pulmonary tuberculosis; $\mathrm{PTB}-=$ smear negative pulmonary tuberculosis; EPTB $=$ extra-pulmonary tuberculosis

$\mathrm{SCC}=$ short course chemotherapy $(2$ months on $\mathrm{RHZ}+/-\mathrm{E}$ or $\mathrm{S}$, followed by 6 months on $\mathrm{EH}$ or $\mathrm{RH})$

$\mathrm{LCC}=$ long course chemotherapy ( 2 months on $\mathrm{EH}+/-\mathrm{S}$, followed by 10 months on $\mathrm{EH}$; TH had also been used as an alternative to $\mathrm{EH}$ until its recent withdrawal).

$\mathrm{R}=$ Rifampicin,; $\mathrm{H}=$ Isoniazid; $\mathrm{Z}$ = Pyrazinamide; $\mathrm{E}=$ Ethambutol; $\mathrm{S}=$ Streptomycin; $\mathrm{T}$ = Thioacetazone.

month and end of treatment smear-positivity (treatment failure), than those on SCC ( $<<0.05$; table 4$)$.

\section{Treatment results}

We analysed treatment outcomes for the years 1994-2000 and, as a result, we evaluated 16943 ( $85 \%$ of the total) cases. Of these, $8268(49 \%)$ successfully completed treatment, $3151(18.6 \%)$ defaulted, 110(0.6\%) had treatment failure, $446(2.6 \%)$ died and $2000(10 \%)$ were transferred out, while $2968(17.5 \%)$ had no record of treatment outcomes. Assuming that patients with unrecorded treatment outcome had all defaulted from treatment, a total of 6119 (36.1\%) patients defaulted.

Among new PTB+ (and all TB) patients, treatment success was higher among patients on SCC, while default and failure rates were higher among those on LCC (Table 5). Death in all categories was higher among those on SCC. Among new cases on SCC, PTB+ cases had 1.8 (65\% vs. $37 \%)$ and $1.4(65 \%$ vs. $48 \%)$ times higher rate of treatment success compared to PTB- and EPTB cases, respectively $(\mathrm{p}<0.001)$. Female PTB+ patients had significantly higher treatment success $(58 \%$ vs. $54 \% ; \mathrm{p}=0.001)$ and lower defaulter rate ( $26 \%$ vs. $30 \%$; $p<0.001)$ than males.

Patients registered as "return after default", had significantly lower treatment success compared to new cases, both for all TB (34\% vs. $49 \%$; $\mathrm{p}<0.001)$, and for PTB+ (34\% vs. 58\%; p < 0.001). Furthermore, return after default cases were more likely to default again compared to new cases among PTB+ patients (53\% vs. $29 \%$; p < 0.001 ) and all TB patients (53\% vs. $36 \%$; p < 0.001 ). Treatment failure was five times higher among cases that received re-treatment for previous failure compared to new cases of PTB+ $(6.4 \%$ vs. $1.2 \%$; $p<0.001)$; however, both groups had comparable treatment success, mainly due to a lower defaulter rate among failure cases ( $24 \%$ vs. $29 \%)$.

Patients for whom follow-up sputum smear remained positive at the second month during treatment had significantly lower treatment success than those with negative smear result ( $53 \%$ vs. $74 \%$; $<0.001)$, mainly as a result of higher defaulter $(24 \%$ vs. $18 \%)$ and failure $(10 \%$ vs. $1 \%)$ rates among the former group.

Table 6 presents treatment outcomes for new cases of TB across the years. A steady rise in treatment success and decline in defaulter rate was noticed particularly among patients on SCC. Treatment failure showed a remarkable decline in both SCC and LCC groups. Death rate did not show much variation across the years.

When we adjusted the outcome measures for treatment results by various potentially confounding variables, significantly higher treatment success was exhibited among female patients, those 15-24 years, patients treated with SCC, those on re-treatment for relapse, PTB+ cases, those treated during 2000 and those treated in the Lemmo district health facilities (Table 7). Meanwhile, male patients, 
Table 6: Treatment outcomes across the years for new cases of tuberculosis

\begin{tabular}{|c|c|c|c|c|c|c|c|}
\hline & 1994 n (\%) & 1995 n (\%) & 1996 n (\%) & 1997 n (\%) & 1998 n (\%) & 1999 n (\%) & 2000 n (\%) \\
\hline \multicolumn{8}{|l|}{ PTB+ on SCC } \\
\hline Treatment success & $5(42)$ & $40(34)$ & $45(38)$ & $122(47)$ & $338(60)$ & $811(69)$ & $805(74)$ \\
\hline Default* & $5(42)$ & $6 I(5 I)$ & $50(42)$ & $77(30)$ & II 4 (20) & 225 (19) & $194(17)$ \\
\hline Failure & - & $3(3)$ & $2(2)$ & $2(1)$ & - & $8(1)$ & $10(1)$ \\
\hline Death & - & $4(3)$ & $2(2)$ & $10(4)$ & $24(4)$ & $44(4)$ & $33(3)$ \\
\hline Transferred out & $2(17)$ & II (9) & $20(17)$ & $46(18)$ & $89(16)$ & $88(7)$ & $50(5)$ \\
\hline \multicolumn{8}{|l|}{ PTB+ on LCC } \\
\hline Treatment success & $188(38)$ & $384(48)$ & $564(56)$ & $27 \mid(45)$ & $308(52)$ & $231(72)$ & $40(68)$ \\
\hline Default* & $190(38)$ & $298(38)$ & $304(30)$ & $246(40)$ & $240(40)$ & $72(22)$ & $15(25)$ \\
\hline Failure & $23(5)$ & $18(2)$ & $8(1)$ & $8(1)$ & $6(1)$ & $3(1)$ & - \\
\hline Death & $17(3)$ & $21(3)$ & $18(2)$ & $20(3)$ & $19(3)$ & $9(3)$ & $3(5)$ \\
\hline Transferred out & $78(16)$ & $71(9)$ & $107(\mathrm{II})$ & $63(10)$ & $24(4)$ & $6(2)$ & I (2) \\
\hline \multicolumn{8}{|l|}{ PTB- on SCC } \\
\hline Treatment success & I (8) & $15(23)$ & $18(20)$ & $32(29)$ & $67(30)$ & 153 (39) & $189(47)$ \\
\hline Default* & $8(67)$ & $40(60)$ & $67(74)$ & $55(50)$ & $85(38)$ & $116(29)$ & $112(28)$ \\
\hline Death & I (8) & $6(9)$ & $2(2)$ & $10(9)$ & $8(4)$ & $25(6)$ & $20(5)$ \\
\hline Transferred out & $2(17)$ & $5(8)$ & $3(3)$ & $12(I I)$ & $65(29)$ & $101(26)$ & $81(20)$ \\
\hline \multicolumn{8}{|l|}{ PTB- on LCC } \\
\hline Treatment success & $107(44)$ & $88(3 I)$ & $84(34)$ & $78(3 I)$ & $199(37)$ & $182(50)$ & $34(47)$ \\
\hline Default* & $101(42)$ & $163(57)$ & $127(5 \mathrm{I})$ & $|4|(57)$ & $280(5 \mathrm{I})$ & $135(37)$ & $25(34)$ \\
\hline Death & $4(2)$ & $5(2)$ & $6(2)$ & $5(2)$ & $16(3)$ & $20(6)$ & I (I) \\
\hline Transferred out & $30(12)$ & $30(\mathrm{II})$ & $31(13)$ & $27(\mathrm{II})$ & $48(9)$ & $25(7)$ & $13(18)$ \\
\hline \multicolumn{8}{|l|}{ EPTB on SCC } \\
\hline Treatment success & $4(2 I)$ & $19(26)$ & $25(44)$ & $29(32)$ & $43(44)$ & $10 \mid(5 I)$ & $215(56)$ \\
\hline Default* & II (58) & $43(59)$ & $29(5 \mathrm{I})$ & $30(33)$ & $29(30)$ & $68(35)$ & $110(28)$ \\
\hline Death & $2(11)$ & $6(8)$ & I (2) & $3(4)$ & I (I) & $4(2)$ & $7(2)$ \\
\hline Transferred out & $2(11)$ & $5(7)$ & $2(4)$ & $28(31)$ & $24(25)$ & $24(12)$ & $53(14)$ \\
\hline \multicolumn{8}{|l|}{ EPTB on LCC } \\
\hline Treatment success & $80(35)$ & $274(49)$ & $559(52)$ & $387(36)$ & $34 I(34)$ & $222(43)$ & $69(50)$ \\
\hline Default* & $122(52)$ & $203(36)$ & $321(30)$ & 412 (39) & $553(56)$ & $253(49)$ & $58(42)$ \\
\hline Death & $2(1)$ & $3(1)$ & $3(0.3)$ & $7(1)$ & $8(1)$ & $14(3)$ & - \\
\hline Transferred out & $28(12)$ & 85 (15) & $198(18)$ & $260(24)$ & $91(9)$ & $24(5)$ & II (8) \\
\hline
\end{tabular}

* Patients with missing information on treatment outcome were assumed to have defaulted and analysed as defaulters.

$\mathrm{PTB}+=$ smear positive pulmonary tuberculosis; $\mathrm{PTB}-=$ smear negative pulmonary tuberculosis; EPTB = extra-pulmonary tuberculosis

SCC $=$ short course chemotherapy ( 2 months on RHZ+/- E or S, followed by 6 months on $\mathrm{EH}$ or $\mathrm{RH}$ )

$\mathrm{LCC}=$ long course chemotherapy ( 2 months on $\mathrm{EH}+/-\mathrm{S}$, followed by 10 months on $\mathrm{EH}$; $\mathrm{TH}$ had also been used as an alternative to $\mathrm{EH}$ until its recent withdrawal).

$\mathrm{R}=$ Rifampicin,; $\mathrm{H}=$ Isoniazid; $\mathrm{Z}=$ Pyrazinamide; $\mathrm{E}=$ Ethambutol; $\mathrm{S}=$ Streptomycin; $\mathrm{T}=$ Thioacetazone.

patients aged 45-54, PTB- cases, those on LCC, return after default cases and those treated in Duna district health facilities exhibited significantly higher default rate.

\section{Trend over time}

DOTS was initiated in 1996 in a hospital and one health centre, with potential population coverage of $25 \%$ (defined as population living within 2 hours walking distance from a health facility; estimated at 250,000 for the hospital and 25000 for the health centre). The number of health facilities providing DOTS increased to 10 in 1997, 30 in 1999 and 41 in 2001, making the population coverage by DOTS $31 \%, 58 \%$ and $75 \%$ respectively. The proportion of patients treated with SCC increased from $7 \%$ in 1994 to $27 \%$ in 1998, 58\% in 1999, and $97 \%$ in 2001. $95 \%(39 / 41)$ of the treatment centres had at least isoniazid, rifampicin, pyrazinamide and ethambutol at the time of visit for data collection. Reagents for Acid-fast stain were available in nine of the 13 diagnostic centres functioning at the time. 
Table 7: Adjusted odds ratios for various factors that might affect treatment outcomes among registered tuberculosis patients

\begin{tabular}{|c|c|c|c|c|c|c|}
\hline \multirow[b]{2}{*}{ Characteristic } & \multicolumn{3}{|c|}{ Treatment success (all TB) } & \multicolumn{3}{|c|}{ Default (all TB) } \\
\hline & Percent ${ }^{1}$ & Adjusted OR* $(95 \% \mathrm{Cl})$ & $\mathrm{p}$-value & Percent ${ }^{1}$ & Adjusted OR* $(95 \% \mathrm{Cl})$ & $\mathrm{p}$-value \\
\hline \multicolumn{7}{|l|}{ Sex } \\
\hline Male & 45.5 & 1.00 & & 37.5 & 1.00 & \\
\hline Female & 49.4 & $1.15(1.08-1.23)$ & $<0.001$ & 34.0 & $0.88(0.82-0.94)$ & $<0.001$ \\
\hline \multicolumn{7}{|l|}{ Age group (years) } \\
\hline $0-14$ & 42.6 & $0.83(0.75-0.92)$ & $<0.001$ & 41.2 & $1.17(1.06-1.30)$ & 0.002 \\
\hline 15-24 (reference group) & 51.1 & 1.00 & & 33.4 & 1.00 & \\
\hline $25-34$ & 47.5 & $0.84(0.77-0.91)$ & $<0.001$ & 34.4 & $1.07(0.98-1.17)$ & 0.14 \\
\hline $35-44$ & 45.7 & $0.76(0.68-0.84)$ & $<0.001$ & 36.1 & $1.14(1.03-1.28)$ & 0.02 \\
\hline $45-54$ & 44.9 & $0.69(0.60-0.79)$ & $<0.001$ & 37.7 & $1.26(1.09-1.46)$ & 0.002 \\
\hline $55-64$ & 51.4 & $1.04(0.84-1.29)$ & 0.71 & 35.4 & $1.04(0.8 \mathrm{I}-1.27)$ & 0.90 \\
\hline$\geq 65$ & 37.4 & $0.67(0.48-0.93)$ & 0.02 & 43.7 & $1.27(0.92-1.726$ & 0.15 \\
\hline \multicolumn{7}{|l|}{ Patient category } \\
\hline New & 47.0 & 1.00 & & 35.9 & 1.00 & \\
\hline Transferred-in & 59.5 & $1.09(0.89-1.36)$ & 0.40 & 35.7 & $1.19(0.95-1.48)$ & 0.13 \\
\hline Return after default & 33.9 & $0.68(0.5 \mathrm{I}-0.92)$ & 0.01 & 52.9 & $1.57(1.19-2.07)$ & 0.002 \\
\hline Treatment failure & 54.3 & $1.30(0.91-1.86)$ & 0.15 & 23.6 & $0.72(0.48-1.09)$ & 0.12 \\
\hline Relapse & 62.9 & $1.51(1.01-2.26)$ & 0.05 & 16.9 & $0.50(0.30-0.82)$ & 0.006 \\
\hline \multicolumn{7}{|l|}{ TB type } \\
\hline Pulmonary positive & 55.3 & 1.00 & & 28.7 & 1.00 & \\
\hline Pulmonary negative & 37.5 & $0.55(0.50-0.60)$ & $<0.001$ & 43.7 & $2.06(1.87-2.26)$ & $<0.001$ \\
\hline Extra-pulmonary & 42.4 & $0.51(0.47-0.55)$ & $<0.001$ & 41.1 & $1.94(1.78-2.11)$ & $<0.001$ \\
\hline \multicolumn{7}{|l|}{ Treatment regimen } \\
\hline Short course chemotherapy & 55.5 & 1.00 & & 27.7 & 1.00 & \\
\hline Long course chemotherapy & 45.0 & $0.68(0.62-0.75)$ & $<0.001$ & 40.9 & $1.46(1.33-1.61)$ & $<0.001$ \\
\hline \multicolumn{7}{|l|}{ Treatment Centre ${ }^{* *}$} \\
\hline Hossana Hospital & 27.0 & 1.00 & & 40.0 & 1.00 & \\
\hline Lemmo district HF & 60.4 & $5.14(4.62-5.72)$ & $<0.001$ & 20.6 & $0.28(0.25-0.31)$ & $<0.001$ \\
\hline Shashogo district HF & 50.8 & $2.8 \mathrm{I}(2.4 \mathrm{I}-3.28)$ & $<0.001$ & 44.1 & $1.18(1.01-1.37)$ & 0.04 \\
\hline Misha district HF & 60.3 & $4.78(4.23-5.39)$ & $<0.001$ & 33.0 & $0.58(0.5 \mathrm{I}-0.65)$ & $<0.001$ \\
\hline Gibe district HF & 60.7 & $4.78(4.20-5.45)$ & $<0.001$ & 26.2 & $0.5 \mathrm{I}(0.45-0.59)$ & $<0.001$ \\
\hline Soro district HF & 50.5 & $2.70(2.39-3.06)$ & $<0.001$ & 42.5 & $1.11(0.98-1.26)$ & 0.09 \\
\hline Duna district HF & 30.2 & $0.77(0.55-1.08)$ & 0.12 & 67.3 & $5.18(3.7 \mid-7.24)$ & $<0.001$ \\
\hline Badewacho district HF & 41.1 & $1.64(1.44-1.86)$ & $<0.001$ & 54.4 & $2.11(1.86-2.40)$ & $<0.001$ \\
\hline \multicolumn{7}{|l|}{ Year of treatment } \\
\hline 1994 & 38.4 & 1.00 & & 42.7 & 1.00 & \\
\hline 1995 & 40.6 & $1.18(0.99-1.39)$ & 0.06 & 39.9 & $1.17(1.00-1.37)$ & 0.05 \\
\hline 1996 & 48.7 & $1.29(1.10-1.5 \mathrm{I})$ & 0.002 & 33.3 & $0.83(0.7 \mathrm{I}-0.97)$ & 0.02 \\
\hline 1997 & 38.9 & $0.72(0.6 \mathrm{I}-0.85)$ & $<0.001$ & 39.5 & $1.08(0.93-1.27)$ & 0.32 \\
\hline 1998 & 41.9 & $0.74(0.63-0.87)$ & $<0.001$ & 43.8 & $1.20(1.03-1.40)$ & 0.02 \\
\hline 1999 & 56.7 & $1.37(1.16-1.62)$ & $<0.001$ & 30.0 & $0.59(0.5-0.70)$ & $<0.001$ \\
\hline 2000 & 59.8 & $1.41(1.18-1.69)$ & $<0.001$ & 25.8 & $0.54(0.45-0.65)$ & $<0.001$ \\
\hline
\end{tabular}

$\mathrm{OR}=$ odds ratio; $\mathrm{Cl}=$ confidence intervals; $\mathrm{HF}=$ Health facilities

* All the variables in the table are included in the model.

** All treatment centres in a district grouped together as a unit.

I Percentage out of total registered for treatment. Patients with missing outcome record were assumed to have defaulted and analysed as defaulters.

Simultaneously, treatment success for new PTB+ patients (on SCC and LCC together) increased from 38\% in 1994 to $56 \%$ in $1998,70 \%$ in 1999 and $73 \%$ in $2000\left(\chi_{\text {trend }^{\prime}} \mathrm{p}\right.$ $<0.001)$. Defaulting among new PTB+ patients declined from $38 \%$ in 1994 to $30 \%$ in $1998,20 \%$ in 1999 and $18 \%$ in $2000\left(\chi_{\text {trend }}^{2} \mathrm{p}<0.001\right)$. Treatment failure decreased from $5 \%$ in 1994 to $1 \%$ in 2000 . The proportion of reported deaths remained unchanged over years with some variations in the range of $2-5 \%$.

\section{Treatment at small and large centres}

Thirty-one out of $40(77.5 \%)$ peripheral treatment centres had significantly higher treatment success, and 28 (70\%) of them had significantly lower defaulter rate compared to 
the zonal hospital (details not shown). Further comparison by districts revealed that six of the seven rural districts had significantly better treatment success while three out of seven had lower defaulter rate than the zonal hospital (Table 7).

\section{Discussion}

The results of our study show that there has been a continuous increase in treatment success and eventual decline in defaulter and failure rates in parallel to the expansion and decentralisation of DOTS to the lower treatment units. Treatment with SCC increased from a very low coverage in 1994 to almost full coverage in 2001 partly due to the expansion of DOTS (with SCC as one of its elements), and partly due to the regional TLCP commitment to SCC both in DOTS and non-DOTS areas. However, trends in case detection and notification showed inconsistencies over years.

In the hierarchy of study designs, observational studies come after the randomised controlled trials [17]. Nevertheless, in the real field condition, where randomised trials are not logistically feasible, such observational study designs may provide important and valid information. Our study design does not allow comparison of treatment outcomes between the DOTS and non-DOTS areas. However, this study gives us a valuable information on the program performance before and along the course of expanding DOTS in this resource-constrained rural setting.

Four years after the introduction of DOTS in the zone, it has been possible to achieve treatment success of $73 \%$ in 2000 for new PTB+ patients. Faring well towards the WHO/IUATLD recommended $85 \%$, this confirms the finding of other studies $[7,18]$ that the DOTS strategy works well in resource-constrained settings with low overall health coverage. Increased coverage by SCC, improved access to care through decentralisation of the service and improved patient follow-up with the introduction of DOTS have most likely played a significant role in improving the treatment outcomes.

Higher treatment success and lower default among female patients confirms previous study findings [11]. However, the proportion of females among patients registered for TB treatment was found to be consistently low, and it was exceptionally lower among patients older than 45 years. This may reflect a genuine gender difference in the TB epidemiology [19-21]. However, the possibility of this being a reflection of gender differentials in access to health care within the society may need to be ruled out by further studies.
Patients treated at the peripheral treatment centres exhibited better treatment outcomes compared to those treated in the zonal hospital, which implies better follow-up of cases or better access to the TB care services.

Despite significant decline in defaulter rate over time, patients that received re-treatment as "return after default", were much more likely to default again compared to new patients. This group of defaulters seems refractory to the conventional approach of treatment supervision; social and cultural factors that might play a role need to be explored. More death among patients on SCC was an indication of the policy that severely sick patients and those co-infected with HIV be a priority for treatment by SCC and these groups were more likely than others to die. Though lower among patients on SCC, the high proportion of failure among patients retreated for previous failure might signal the emergence of multi-drug resistant TB (MDR-TB). Earlier studies have shown that MDR has so far been below $1 \%[22,23]$.

The proportion of $\mathrm{PTB}+$ was fairly stable in the range of $40-50 \%$ across the years, which indicates that the diagnosticians uniformly observed the diagnostic algorithms recommended by the NTLCP. The case detection rate (CDR), as estimated by the proportion of expected incident cases notified, showed an encouraging increase from $45 \%$ in 1994 to $115 \%$ in 1999 , well above the CDR recommended by the WHO. The most plausible explanation for this might be that the vigorous implementation of the program during the earlier years of introduction of DOTS might have enabled detection of a big pool of prevalent cases of TB. But the pace in case detection could not be maintained during 2000 and 2001 when the CDR fell to $94 \%$ and $67 \%$ respectively. Reduction in the incidence of TB could be a possible explanation, but one cannot justify such a fast decline in a very short time. This calls for some case detection improvement initiatives to be in place as the proportion of $\mathrm{TB}$ cases detected and cured under DOTS is one of the health-related indicators of the millennium development goals [24]. Quality of the laboratory services and the possibility of under-reporting are among factors to be explored further.

Two major areas of program weakness need to be addressed. First, 94\% of the patients were registered as new cases, which suggests that some re-treatment cases had been wrongly classified as new. Further, treatment outcomes have not been recorded for about one-fifth of the patients. Improving the record keeping system seems to be an immediate priority. Second, the proportion of $\mathrm{PTB}+$ patients who had follow-up sputa examined for AFB was low. The threat of anti-TB drug resistance is imminent, and it is important to strengthen patient follow-up with sputum examinations. About one-third of the 


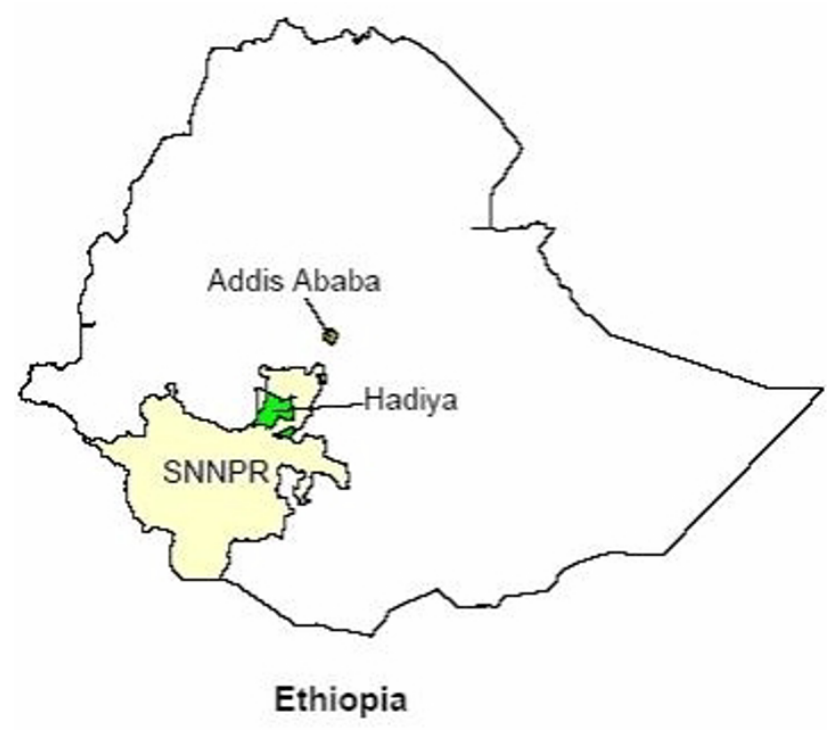

Figure I

Map of Ethiopia with the study area highlighted. Ethiopia is administratively divided into nine regional states and two city administrations, and the Southern Nations, Nationalities and Peoples' Regional State (SNNPRS) accounts for one-fifth (I 3 million) of the total population of the country. The study area (Hadiya zone) has got a population of I.2 million.

peripheral diagnostic facilities lacked reagents for ZiehlNeelsen stain during the time of visit, and this might partly explain the low performance.

\section{Conclusion}

The introduction and expansion of DOTS in Hadiya has led to a significant increase in treatment success and decrease in default and failure rates. The smaller institutions exhibited better treatment outcomes compared to the larger ones including the zonal hospital. The high number of patients with missing information in the unit registers is perhaps an issue that needs to be addressed as urgently as possible. Further studies are recommended to see the impact of the programme on the prevalence and incidence of tuberculosis.

\section{Competing interests}

The author(s) declare that they have no competing interests.

\section{Authors' contributions}

EBS was the principal investigator and participated in the design of the study, conducted the study, performed data entry and analysis, and wrote the manuscript. BL was the project co-ordinator and participated in the design, data analysis and write-up of the manuscript.

\section{Acknowledgements}

We would like to thank the Southern Nations, Nationalities and Peoples' Regional Health Bureau, the regional and zonal TLCP and the staff of respective health facilities involved in the study for their genuine support during the field data collection. The Centre for International Health, University of Bergen funded this study.

\section{References}

I. Dye C, Scheele S, Dolin P, Pathania V, Raviglione MC: Consensus statement. Global burden of tuberculosis: estimated incidence, prevalence, and mortality by country. WHO Global Surveillance and Monitoring Project. JAMA 1999, 282:677-686.

2. World Health Organization: 5050:Towards a TB-free future. Geneva, WHO; 200I.

3. Southern Ethiopia Regional State Health Bureau: The Health Sector Development Plan (2000-2004). Awassa, RHB; 200I.

4. Lindtjørn B, Madebo $T$ : The outcome of tuberculosis treatment at a rural hospital in Southern Ethiopia. Tropical Doctor 200I, 3 I: I32-135.

5. Yassin MA, Takele L, Gebresenbet S, Girma E, Lera M, Lendebo E, Cuevas LE: HIV and Tuberculosis Coinfection in the Southern Region of Ethiopia: A Prospective Epidemiological Study. Scand J Infect Dis 2004, 36:670-673.

6. Pio A, Luelmo F, Kumaresan J, Spinaci S: National tuberculosis program review: experience over the period 1990-95. Bull World Health Organ 1997, 75:569-58I.

7. Zhang L-X, Tu D H, Enarson D A: The impact of directly observed treatment on the epidemiology of tuberculosis in Beijing. Int J Tuberc lung Dis 2000, 4:904-910.

8. Kazeonny B, Khorosheva T, Aptekar T, Rybka L, Kluge H, Jakubowiak W, Pashkevich D: Evaluation of Directly Observed Therapy Short Course strategy for treating tuberculosis- Orel Oblast, Russian Federation, I 999-2000. MMWR 200I, 50:204-206.

9. Kassim S, Sassam-Morokro M, Akhan A, Abouya L Y, Digbeu H, Yesso G, Coulibaly I M, Coulibaly D, Whitaker P J DR, et al: Two-year follow-up of persons with HIV-I and HIV-2-associated pulmonary tuberculosis treated with short-course in West Africa. AIDS (London, England) 1995, 9: I 185-1191.

10. Volmink J, Garner P: Directly observed therapy for treating tuberculosis (Chocrane Review). Oxford, The Chocrane Library; 200I.

II. Wally JD, Khan MA, Newell JN, Khan MH: Effectiveness of the direct observation component of DOTS for tuberculosis: a randomised controlled trial in Pakistan. Lancet 200I, 357:664-669.

12. Kelly P M, Cumming R G, Kaldor J M: HIV and tuberculosis in rural sub-Saharan Africa: a cohort study with two-year follow-up. Transactions of the Royal Society of Tropical Medicine and Hygiene 1999, 93:287-293.

13. Central Statistics Authority: The $\mathbf{1 9 9 4}$ Population and Housing Census of Ethiopia: Results for the Southern Nations, Nationalities and People's Region. Addis Ababa, CSA; 1996.

14. Hadiya zonal department of Planning and Economic Development: The popolation of Hadiya zone by woredas: projected from the results of 1994 Population and Housing Census. Hossana, ; 2002.

15. Ministry of Health of Ethiopia: Tuberculosis and Leprosy Control Programme Manual. 2nd edition. Addis Ababa, MOH; 2002.

16. SPSS for windows. Release II.0.0 (19 Sep 200I). Standard Version. , SPSS inc.; 200I.

17. Grimes DA, Schulz KF: An overview of clinical research: the lay of the land. Lancet 2002, 359:57-6I.

18. Balasubramanian V N, Oommen K, Samuel R: DOT or not? Direct observation of anti-tuberculosis treatment and patient outcomes, Kerala State, India. Int J Tuberc lung Dis 2000, 4:409-4I 3.

19. Holmes C B, Hausler H, Nunn P: A review of sex differences in the epidemiology of tuberculosis. Int J Tuberc lung Dis 1998, 2:96-104. 
20. Hamid Salim M A, Declercq E, Van Deun A, Saki K A R: Gender differences in tuberculosis: a prevalence survey done in Bangladesh. Int J Tuberc lung Dis 2004, 8:952-957.

21. Balasubramanian R, Garg R, Santha T, Gopi P G, Subramani R, Chandrasekaran V, Thomas A, Rajeswari R, Ananadakrishnan S, Perumal M, Niruparani C, Sudha G, Jaggarajamma J, Frieden T R, Narayanan P N: Gender disparities in tuberculosis: report from a rural DOTS programme in south India. Int J Tuberc lung Dis 2004, 8:323-332.

22. Demissie M, Gebeyehu M, Berhane Y: Primary resistance to antituberculosis drugs in Addis Ababa, Ethiopia. Int J Tuberc lung Dis 1997, I:64-67.

23. Demissie M, Lemma E, Gebeyehu M, Lindtjørn B: Sensitivity to Anti-tuberculosis Drugs in HIV-positive and -negative Patients in Addis Ababa. Scand J Infect Dis 200I, 33:914-919.

24. World Health Organization: The World Health Report 2003: Shaping the Future. Geneva, WHO; 2003.

\section{Pre-publication history}

The pre-publication history for this paper can be accessed here:

http://www.biomedcentral.com/1471-2458/5/62/prepub

Publish with Biomed Central and every scientist can read your work free of charge

"BioMed Central will be the most significant development for disseminating the results of biomedical research in our lifetime. "

Sir Paul Nurse, Cancer Research UK

Your research papers will be:

- available free of charge to the entire biomedical community

- peer reviewed and published immediately upon acceptance

- cited in PubMed and archived on PubMed Central

- yours - you keep the copyright

Submit your manuscript here:

http://www.biomedcentral.com/info/publishing_adv.asp 